Recent Insights into the Physics of the Sun and Heliosphere:

Highlights from SOHO and Other Space Missions

IAU Symposium, Vol. 203, 2001

P. Brekke, B. Fleck, and J. B. Gurman eds.

\title{
A Cylindrical Formulation of Force Free Magnetic Fields
}

E. A. Evangelidis

Centre for Physics and Mathematics, 2 Solonos Street, Volos TK38221, Greece

G. J. J. Botha

Department of Mathematics, University College London, Gower Street, London WC1E 6BT, United Kingdom

Abstract. A new solution of the force free field equation is presented in cylindrical coordinates.

\section{Introduction}

The concept of magnetic configurations producing no effect on ionised matter was introduced in astrophysics by Lüst and Schlüter (1954) in order to interpret an eruption on the Sun. The explicit solution of the governing equation

$$
\nabla \wedge \mathbf{B}=\alpha \mathbf{B}
$$

was given by Chandrasekhar (1956), Chandrasekhar and Kendall (1957) in terms of the Hansen (1935) solution of the vectorial Helmholtz equation:

$$
\nabla^{2} \mathbf{B}+k^{2} \mathbf{B}=0 .
$$

The rotation of (1) leads to (2) for divergence free fields. It follows that all solutions of the force free field equation should be members of the family of solutions to the vectorial Helmholtz equation, although the reverse is not true. Following previous work where the integral of energy is presented for Arnold's solution (Arnold 1965) of the force free equation (Evangelidis, Vaughan, \& Botha 2000), a cylindrical formulation of Hansen's solution is given here.

\section{The solution for cylindrical magnetic tubes}

The vectorial Helmholtz equation (2) has vector solutions (Hansen 1935) in the form

$$
\mathbf{L}=\nabla \psi, \mathbf{M}=\nabla \wedge(\psi \mathbf{a}), \mathbf{N}=\nabla \wedge \nabla \wedge(\psi \mathbf{a})
$$

subject to the scalar function $\psi$ satisfying the scalar equation

$$
\nabla^{2} \psi+k^{2} \psi=0
$$

It is noticed that the gradient solution $\mathbf{L}$ in (3) is not a solution of equation (1) although it is a solution of $\mathbf{B} \wedge \nabla \wedge \mathbf{B}=0$. Although the constant vector a can 

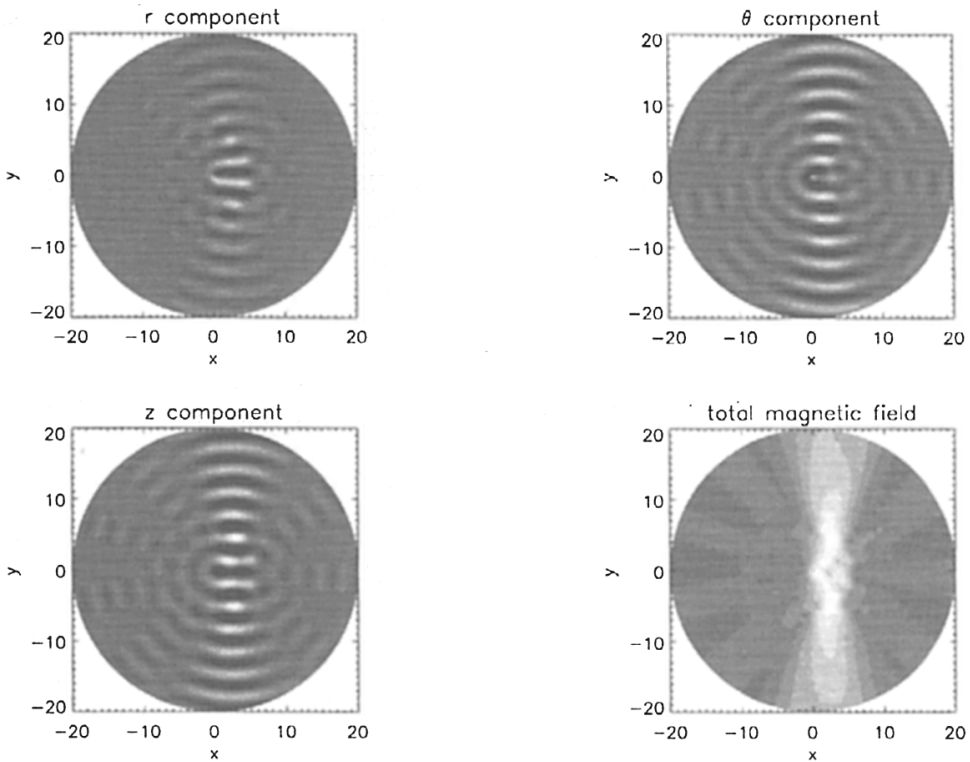

Figure 1. Contour plots of the magnetic field and its components at position $z=3$ along the cylindrical axis. The minimum value of -3 is coded in black, while the maximum is +3 and is coded in white. Zero is coded in grey.
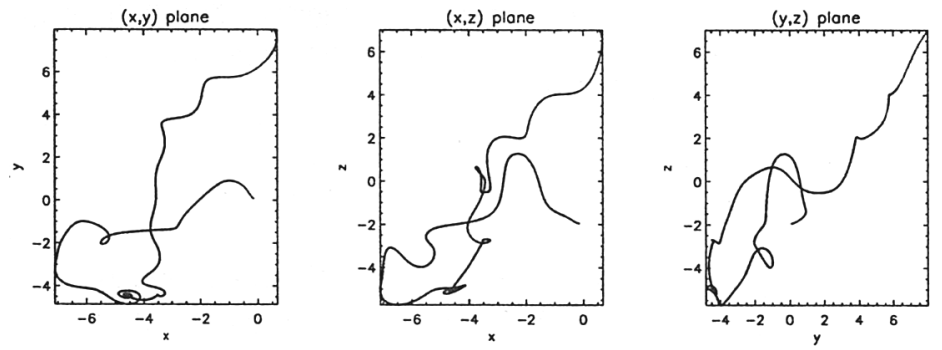

Figure 2. Tracing a magnetic field line from $\left(r_{0}, \theta_{0}, z_{0}\right)=(8,1.5,7)$ at $t=0$ until $t_{\text {fin }}=267$ when it approaches $r=0$. The field line is presented in Cartesian space to correspond with Figure 1. 
be any arbitrary direction in Cartesian coordinates, we have found that this freedom of choice in cylindrical coordinates is reduced to a single direction: that of $\mathbf{e}_{z}$. We have also found that this method of constructing solutions fails completely in toroidal coordinates. For divergence free magnetic fields, the solution is given by the combination

$$
\mathbf{B}=\nabla \wedge(\psi \mathbf{a})+k^{-1} \nabla \wedge \nabla \wedge(\psi \mathbf{a}) .
$$

A similar description of the magnetic field was used to interpret simulations of solar prominence eruptions in spheromacs (Bellan \& Hansen 1998).

Consider the disturbance $\exp \imath\left(k_{0} z+m \theta\right)$ in cylindrical coordinates. The radial dependence of equation (4) then gives the Bessel equation with solutions the Bessel functions $J_{m}(K r)$ and $Y_{m}(K r)$, where $K^{2}=k^{2}-k_{0}^{2}$. Results are presented for the interior solution only, with $C_{m}$ the constants of integration. Analogous expressions can be obtained for the exterior solution by replacing the symbols $J_{m}$ with the symbols $Y_{m}$.

$$
\begin{aligned}
& B_{r}=-K \sum_{m=0}^{\infty} C_{m}\left[\frac{m}{K r}\left(1+\frac{k_{0}}{k}\right) J_{m}-\frac{k_{0}}{k} J_{m+1}\right] \sin \left(k_{0} z-m \theta\right) \\
& B_{\theta}=-K \sum_{m=0}^{\infty} C_{m}\left[\frac{m}{K r}\left(1+\frac{k_{0}}{k}\right) J_{m}-J_{m+1}\right] \cos \left(k_{0} z-m \theta\right) \\
& B_{z}=\frac{K^{2}}{k} \sum_{m=0}^{\infty} C_{m} J_{m} \cos \left(k_{0} z-m \theta\right)
\end{aligned}
$$

Solutions (6) of the force free magnetic fields in cylindrical coordinates, are drawn in Figure 1 for the axial value $z=3$ and $C_{m}=1$ for $m=1,2,3, \ldots 10$. A contour plot of each component of the field is shown, as well as the total field size $B=\sqrt{B_{r}^{2}+B_{\theta}^{2}+B_{z}^{2}}$. The magnetic field lines can be traced by using solutions (6) in

$$
d t=\frac{d r}{B_{r}}=\frac{r d \theta}{B_{\theta}}=\frac{d z}{B_{z}} .
$$

An initial value in the cylinder is chosen and the field line is followed numerically using a fourth order Runge-Kutta method to integrate (7). Some typical results are shown in Figure 2. The direction as well as the speed at which the field line is traced vary during the simulation.

\section{References}

Arnold, V. I. 1965, Comptes Rendues de l'Academie des Sciences, Paris, 261, 17 Bellan, P. M., \& Hansen, J. F. 1998, Physics of Plasmas, 5, 1991

Chandrasekhar, S. 1956, Proc. Nat. Acad. Sci., 42, 1

Chandrasekhar, S., \& Kendall, P. C. 1957, ApJ, 126, 457

Evangelidis, E. A., Vaughan, L. L., \& Botha, G. J. J. 2000, Solar Physics, 193, 17

Hansen, W. W. 1935, Phys.Rev., 47, 139

Lüst, R., \& Schlüter, A. 1954, ZAp, 34, 263 